\title{
Volumetric, Optical, Acoustical and Viscometric Study of Molecular Association in Binary Mixtures of Butylamine with 1-Butanol and Tert-Butanol
}

\author{
S. Singh, S. Parveen, D. Shukla, M. Gupta*
}

AND J.P. SHUKLA

Department of Physics, University of Lucknow, Lucknow 2260007, India

(Received January 15, 2007; revised version April 20, 2007)

Density $(\rho)$, refractive index $(n)$, ultrasonic velocity $(u)$, and viscosity $(\eta)$ were measured for the binary mixtures formed by butylamine with 1-butanol and tert-butanol at temperatures 293, 303, and $313 \mathrm{~K}$ over the entire composition range. Excess molar volume $V_{\mathrm{m}}^{\mathrm{E}}$, molar refraction deviation $\Delta R_{\mathrm{m}}$, deviation in ultrasonic velocity $\Delta u$, viscosity deviation $\Delta \eta$, and excess Gibb's free energy of activation for viscous flow $\Delta G^{* \mathrm{E}}$ were derived from the experimental data and the computed results were fitted to the Redlich-Kister polynomial equation. The values of $V_{\mathrm{m}}^{\mathrm{E}}, \Delta R_{\mathrm{m}}, \Delta u, \Delta \eta$, and $\Delta G^{* \mathrm{E}}$ were plotted against the mole fraction of butylamine. The observed positive and negative values of excess parameters for both the mixtures were explained on the basis of intermolecular interactions present in these mixtures. The higher negative values of $V_{\mathrm{m}}^{\mathrm{E}}$ and $\Delta R_{\mathrm{m}}$ and positive values of $\Delta u, \Delta \eta$, and $\Delta G^{* \mathrm{E}}$ for butylamine +1 -butanol mixture suggest that specific interactions are taking place in butylamine +1 -butanol mixture, while weak dipole-dipole induced forces and dispersive forces seem to be responsible for butylamine + tert-butanol mixture. Furthermore, different empirical relations were used to correlate the binary refractive indices. An excellent agreement was found between experimental and theoretical values.

PACS numbers: 43.35.+d, 62.60.+v, 82.60.Lf

\section{Introduction}

The knowledge of the thermodynamic properties of organic liquid mixtures is very important for understanding the molecular interactions between the com-

*corresponding author; e-mail: guptagm@rediffmail.com 
ponents. This also helps to evolve theoretical models and is useful in industrial applications [1-4]. Excess properties of liquid systems, such as excess molar volume, deviation in molar refraction are needed for the design of separation equipment and to test the theories of the solution [5]. In addition, excess properties provide information about the molecular interactions and macroscopic behaviour of fluid mixtures and can be used to test and to improve thermodynamical models for calculating and predicting the fluid phase equilibria. The knowledge of excess Gibb's free energy of activation for viscous flow helps in understanding the molecular orientation and to study the extent of intermolecular interaction between component molecules of the liquid mixtures. In recent years, there has been considerable upsurge in the theoretical and experimental investigation of the excess thermodynamic properties of binary liquid mixtures [6, 7].

Butylamine is commonly used as an intermediate for the production of pharmaceuticals, dyestuffs, emulsifying agents, insecticides, synthetic tanning agents, and rubber chemicals. Also, butylamine is used as a raw material for herbicides, corrosion inhibitors, vulcanization accelerators, and flotation agents.

Alcohols are self associated organic liquids and are widely used as basic organic compound for the synthesis of other organic compounds; $n$-butylalcohols have been administered to patients for the control of postoperative pain in otolaryngeal surgery and for an unexplained anti-haemorrhagic effect in those with far advanced cancer.

In order to examine molecular interactions in the mixture of butylamine with alcohols through excess properties viz. excess molar volume $V_{\mathrm{m}}^{\mathrm{E}}$, molar refraction deviation $\Delta R_{\mathrm{m}}$, deviation in ultrasonic velocity $\Delta u$, viscosity deviation $\Delta \eta$, and excess Gibb's free energy of activation for viscous flow $\Delta G^{* \mathrm{E}}$, we report here density $(\rho)$, refractive index $(n)$, ultrasonic velocity $(u)$, and viscosity $(\eta)$ of binary mixtures of butylamine with 1-butanol and tert-butanol over the entire composition range at temperatures 293, 303, and $313 \mathrm{~K}$. Further, seven mixing rules for the prediction of refractive index proposed by Lorentz-Lorentz $(\mathrm{L}-\mathrm{L})$, Gladstone-Dale (G-D), Weiner (W), Heller (H), Arago-Biot (A-B), Newton (N), and Eykmen (Eyk) have been theoretically applied to these binary mixtures. A comparative study has been made between the experimental and theoretically calculated values of refractive indices at all the three temperatures. The results have been discussed in terms of average percentage deviation (APD).

\section{Experimental section}

The densities were measured using a pyknometer having a bulb volume of $8 \mathrm{ml}$ and an internal diameter of the capillary of about $0.1 \mathrm{~cm}$ with an accuracy $\pm 0.5 \%$. The pyknometer was calibrated at $298 \mathrm{~K}$ with double distilled water and benzene. The pyknometer with the test solution was equilibrated in a thermostatically controlled water bath maintained at $\pm 0.1 \mathrm{~K}$ of the desired temperature. An average of triplicate measurements was taken into account. 
Refractive index was measured using Abbe's refractometer provided by Optics Technologies, Delhi. The refractometer was calibrated by measuring the refractive indices of triply distilled water and benzene at $T=293 \mathrm{~K}$. The accuracy in the refractive index measurement was 0.001 units. Temperature was controlled by circulating adequately stirred water bath (accuracy $\pm 0.1^{\circ} \mathrm{C}$ ). The sample mixtures were directly injected into the prism assembly of the instrument by means of an air-tight hypodermic syringe. An average of four to five measurements was taken for each sample mixture.

The ultrasonic velocity of pure components and their mixtures were measured by variable path fixed frequency interferometer provided by Mittal Enterprises, New Delhi (Model-83). It consists of a high frequency generator and a measuring cell. The calibration of ultrasonic interferometer was done by measuring the velocity in AR grade benzene and carbon tetrachloride. Standard value of $u$ for benzene and carbon tetrachloride were calculated from the literature value [8] of $u$ at $298 \mathrm{~K}$ and $-\mathrm{d} u / \mathrm{d} t$. Our measured values of $u$ agree closely with the literature values [8]. The maximum estimated error in ultrasonic velocity measurements has been found to be $\pm 0.08 \%$. The measured values of standard deviations of velocities have been found to be smaller than $0.12 \mathrm{~m} / \mathrm{s}$ for the mixtures studied at various temperatures for fine measurements. The temperature was controlled by circulating water around the liquid cell from thermostatically controlled adequately stirred water bath (accuracy $\pm 0.1^{\circ} \mathrm{C}$ ).

The viscosity was measured by Ostwald's viscometer which was calibrated at $298 \mathrm{~K}$ with double distilled water and purified methanol using density and viscosity values from the literature [8]. The flow times were accurate to $\pm 0.1 \mathrm{~s}$, and the uncertainty in the viscosity measurements was within $\pm 0.5 \%$. An average of 3-4 flow times were taken to estimate the viscosity.

Mixtures were prepared by weighing the liquids in specially designed ground glass stopped bottles, taking extreme precautions to minimize preferential evaporation. A Sartorius (BP 121S) single pan balance having a stated precision of $0.1 \mathrm{mg}$ was used throughout. The maximum possible error in the mole fraction is estimated to be \pm 0.0001 . The chemicals used were obtained from Ranbaxy Fine Chemicals Limited. All the chemicals used were purified by standard procedure, discussed by Perrin and Armarego [9]. All the samples were kept in tightly sealed bottles to minimize the absorption of atmospheric moisture.

\section{Results and discussion}

The experimental values of $\rho, n, u$, and $\eta$ at three temperatures viz. 293, 303, and $313 \mathrm{~K}$ are given in Tables I and II for butylamine + 1-butanol and butylamine + tert-butanol mixtures.

The experimental values of $\rho, n, u$, and $\eta$ were used to calculate the values of $V_{\mathrm{m}}^{\mathrm{E}}, \Delta R_{\mathrm{m}}, \Delta u, \Delta \eta$, and $\Delta G^{* \mathrm{E}}$ using the standard relations 
TABLE I

Density $(\rho)$, refractive index $(n)$, ultrasonic velocity $(u)$, viscosity $(\eta)$, excess molar volume $V_{\mathrm{m}}^{\mathrm{E}}$, molar refraction deviation $\Delta R_{\mathrm{m}}$, deviation in ultrasonic velocity $\Delta u$, viscosity deviation $\Delta \eta$, and excess Gibb's free energy of activation for viscous flow $\Delta G^{* \mathrm{E}}$ for mixture butylamine + 1-butanol with mole fractions of butylamine $\left(x_{1}\right)$ at $T=293,303$, and $313 \mathrm{~K}$.

\begin{tabular}{|c|c|c|c|c|c|c|c|c|c|}
\hline$x_{1}$ & $\begin{array}{c}\rho \\
{\left[\mathrm{g} / \mathrm{cm}^{3}\right]}\end{array}$ & $n$ & $\begin{array}{c}u \\
{[\mathrm{~m} / \mathrm{s}]}\end{array}$ & $\left.\begin{array}{c}\eta \\
{[\mathrm{mPa}}\end{array}\right]$ & $\begin{array}{c}V_{\mathrm{m}}^{\mathrm{E}} \\
{[\mathrm{cc} / \mathrm{mol}]}\end{array}$ & $\Delta R_{\mathrm{m}}$ & $\begin{array}{c}\Delta u \\
{[\mathrm{~m} / \mathrm{s}]}\end{array}$ & $\begin{array}{c}\Delta \eta \\
{[\mathrm{mPa} s]}\end{array}$ & $\begin{array}{c}\Delta G^{* \mathrm{E}} \\
{[\mathrm{kJ} / \mathrm{mol}]}\end{array}$ \\
\hline \multicolumn{10}{|c|}{$T=293 \mathrm{~K}$} \\
\hline 0.0000 & 0.8098 & 1.399 & 1286 & 2.8250 & 0.0000 & 0.0000 & 0.00 & 0.0000 & 0.000 \\
\hline 0.1011 & 0.8029 & 1.399 & 1282 & 2.7285 & -0.0600 & -0.0496 & 0.19 & 0.1268 & 0.290 \\
\hline 0.2025 & 0.7969 & 1.400 & 1279 & 2.6276 & -0.1870 & -0.0879 & 0.37 & 0.2500 & 0.571 \\
\hline 0.3028 & 0.7915 & 1.401 & 1275 & 2.5339 & -0.4110 & -0.1217 & 0.51 & 0.3779 & 0.850 \\
\hline 0.4032 & 0.7848 & 1.401 & 1272 & 2.3650 & -0.4630 & -0.1357 & 0.57 & 0.4307 & 1.053 \\
\hline 0.5033 & 0.7780 & 1.401 & 1269 & 2.1510 & -0.4780 & -0.1416 & 0.62 & 0.4379 & 1.193 \\
\hline 0.6032 & 0.7707 & 1.402 & 1265 & 1.9208 & -0.4200 & -0.1205 & 0.56 & 0.4284 & 1.290 \\
\hline 0.7030 & 0.7632 & 1.402 & 1262 & 1.6457 & -0.3160 & -0.1047 & 0.45 & 0.3737 & 1.286 \\
\hline 0.8020 & 0.7558 & 1.402 & 1258 & 1.3375 & -0.2070 & -0.0894 & 0.28 & 0.2842 & 1.151 \\
\hline 0.9000 & 0.7484 & 1.403 & 1255 & 0.9978 & -0.0760 & -0.0391 & 0.15 & 0.1611 & 0.803 \\
\hline 1.0000 & 0.7414 & 1.403 & 1251 & 0.6158 & 0.0000 & 0.0000 & 0.00 & 0.0000 & 0.000 \\
\hline \multicolumn{10}{|c|}{$T=303 \mathrm{~K}$} \\
\hline 0.0000 & 0.8017 & 1.392 & 1228 & 2.2970 & 0.0000 & 0.0000 & 0.00 & 0.0000 & 0.000 \\
\hline 0.1011 & 0.7953 & 1.392 & 1227 & 2.2045 & -0.1280 & -0.0610 & 0.29 & 0.0855 & 0.264 \\
\hline 0.2025 & 0.7893 & 1.393 & 1226 & 2.1168 & -0.3000 & -0.1047 & 0.46 & 0.1765 & 0.530 \\
\hline 0.3028 & 0.7835 & 1.393 & 1224 & 2.0382 & -0.4800 & -0.1473 & 0.64 & 0.2744 & 0.798 \\
\hline 0.4032 & 0.7769 & 1.393 & 1224 & 1.8997 & -0.5460 & -0.1846 & 0.70 & 0.3128 & 0.987 \\
\hline 0.5033 & 0.7700 & 1.393 & 1222 & 1.7283 & -0.5680 & -0.1874 & 0.71 & 0.3177 & 1.116 \\
\hline 0.6032 & 0.7628 & 1.394 & 1221 & 1.5458 & -0.5300 & -0.1719 & 0.69 & 0.3112 & 1.202 \\
\hline 0.7030 & 0.7554 & 1.394 & 1220 & 1.3303 & -0.4570 & -0.1545 & 0.59 & 0.2714 & 1.192 \\
\hline 0.8020 & 0.7480 & 1.394 & 1218 & 1.0910 & -0.3561 & -0.1215 & 0.46 & 0.2064 & 1.057 \\
\hline 0.9000 & 0.7405 & 1.395 & 1216 & 0.8289 & -0.2200 & -0.0547 & 0.29 & 0.1170 & 0.728 \\
\hline 1.0000 & 0.7324 & 1.395 & 1215 & 0.5358 & 0.0000 & 0.0000 & 0.00 & 0.0000 & 0.000 \\
\hline \multicolumn{10}{|c|}{$T=313 \mathrm{~K}$} \\
\hline 0.0000 & 0.7934 & 1.389 & 1189 & 1.8880 & 0.0000 & 0.0000 & 0.00 & 0.0000 & 0.000 \\
\hline 0.1011 & 0.7872 & 1.389 & 1186 & 1.8014 & -0.1770 & -0.0740 & 0.46 & 0.0569 & 0.240 \\
\hline 0.2025 & 0.7813 & 1.389 & 1182 & 1.7252 & -0.3840 & -0.1542 & 0.64 & 0.1247 & 0.491 \\
\hline 0.3028 & 0.7752 & 1.390 & 1178 & 1.6587 & -0.5570 & -0.1940 & 0.79 & 0.2006 & 0.748 \\
\hline 0.4032 & 0.7684 & 1.390 & 1174 & 1.5443 & -0.6200 & -0.2055 & 0.89 & 0.2286 & 0.925 \\
\hline 0.5033 & 0.7614 & 1.390 & 1170 & 1.4071 & -0.6510 & -0.2108 & 0.96 & 0.2335 & 1.045 \\
\hline 0.6032 & 0.7540 & 1.391 & 1166 & 1.2591 & -0.6200 & -0.1970 & 0.92 & 0.2274 & 1.119 \\
\hline 0.7030 & 0.7466 & 1.391 & 1162 & 1.0884 & -0.5540 & -0.1813 & 0.84 & 0.1984 & 1.104 \\
\hline 0.8020 & 0.7390 & 1.391 & 1158 & 0.9004 & -0.4480 & -0.1520 & 0.70 & 0.1509 & 0.972 \\
\hline 0.9000 & 0.7314 & 1.392 & 1154 & 0.6960 & -0.3300 & -0.0810 & 0.47 & 0.0855 & 0.660 \\
\hline 1.0000 & 0.7225 & 1.392 & 1150 & 0.4685 & 0.0000 & 0.0000 & 0.00 & 0.0000 & 0.000 \\
\hline
\end{tabular}

$$
\begin{aligned}
& V_{\mathrm{m}}^{\mathrm{E}}=\frac{x_{1} M_{1}+x_{2} M_{2}}{\rho_{\mathrm{m}}}-\left(\frac{x_{1} M_{1}}{\rho_{1}}+\frac{x_{2} M_{2}}{\rho_{2}}\right), \\
& \Delta R_{\mathrm{m}}=R_{\mathrm{m}}^{\mathrm{expt}}-R_{\mathrm{m}}^{\mathrm{id}},
\end{aligned}
$$

where 
TABLE II

Density $(\rho)$, refractive index $(n)$, ultrasonic velocity $(u)$, viscosity $(\eta)$, excess molar volume $V_{\mathrm{m}}^{\mathrm{E}}$, molar refraction deviation $\Delta R_{\mathrm{m}}$, deviation in ultrasonic velocity $\Delta u$, viscosity deviation $\Delta \eta$, and excess Gibb's free energy of activation for viscous flow $\Delta G^{* \mathrm{E}}$ for mixture butylamine + tert-butanol with mole fractions of butylamine $\left(x_{1}\right)$ at $T=293,303$, and $313 \mathrm{~K}$.

\begin{tabular}{|c|c|c|c|c|c|c|c|c|c|}
\hline$x_{1}$ & $\begin{array}{c}\rho \\
{\left[\mathrm{g} / \mathrm{cm}^{3}\right]}\end{array}$ & $n$ & $\begin{array}{c}u \\
{[\mathrm{~m} / \mathrm{s}]}\end{array}$ & $\left.\begin{array}{c}\eta \\
{[\mathrm{mPa}}\end{array}\right]$ & $\begin{array}{c}V_{\mathrm{m}}^{\mathrm{E}} \\
{[\mathrm{cc} / \mathrm{mol}]}\end{array}$ & $\Delta R_{\mathrm{m}}$ & $\begin{array}{c}\Delta u \\
{[\mathrm{~m} / \mathrm{s}]}\end{array}$ & $\begin{array}{c}\Delta \eta \\
{[\mathrm{mPa} s]}\end{array}$ & $\begin{array}{c}\Delta G^{* \mathrm{E}} \\
{[\mathrm{kJ} / \mathrm{mol}]}\end{array}$ \\
\hline \multicolumn{10}{|c|}{$T=293 \mathrm{~K}$} \\
\hline 0.0000 & 0.8018 & 1.396 & 1208 & 6.6250 & 0.0000 & 0.0000 & 0.00 & 0.0000 & 0.000 \\
\hline 0.1012 & 0.7953 & 1.397 & 1213 & 3.3710 & -0.0003 & -0.0058 & 0.11 & -2.6459 & -1.060 \\
\hline 0.2015 & 0.7890 & 1.398 & 1217 & 2.4141 & -0.0006 & -0.0138 & 0.21 & -3.0000 & -1.292 \\
\hline 0.3026 & 0.7827 & 1.398 & 1221 & 1.8566 & -0.0008 & -0.0192 & 0.28 & -2.9500 & -1.347 \\
\hline 0.4031 & 0.7765 & 1.399 & 1226 & 1.4827 & -0.0009 & -0.0221 & 0.34 & -2.7200 & -1.313 \\
\hline 0.5032 & 0.7704 & 1.400 & 1230 & 1.2412 & -0.0009 & -0.0225 & 0.36 & -2.3600 & -1.166 \\
\hline 0.6029 & 0.7645 & 1.400 & 1234 & 1.0820 & -0.0009 & -0.0205 & 0.36 & -1.9200 & -0.924 \\
\hline 0.7026 & 0.7586 & 1.401 & 1239 & 0.9192 & -0.0008 & -0.0161 & 0.30 & -1.4837 & -0.744 \\
\hline 0.7980 & 0.7586 & 1.402 & 1243 & 0.8050 & -0.0006 & -0.0097 & 0.23 & -1.0246 & -0.515 \\
\hline 0.9012 & 0.7470 & 1.403 & 1247 & 0.7173 & -0.0003 & -0.0021 & 0.11 & -0.4922 & -0.200 \\
\hline 1.0000 & 0.7414 & 1.403 & 1251 & 0.6158 & 0.0000 & 0.0000 & 0.00 & 0.0000 & 0.000 \\
\hline \multicolumn{10}{|c|}{$T=303 \mathrm{~K}$} \\
\hline 0.0000 & 0.7944 & 1.389 & 1180 & 5.5260 & 0.0000 & 0.0000 & 0.00 & 0.0000 & 0.000 \\
\hline 0.1012 & 0.7877 & 1.390 & 1184 & 3.0631 & -0.0003 & -0.0068 & 0.14 & -1.9639 & -0.891 \\
\hline 0.2015 & 0.7812 & 1.390 & 1187 & 2.2105 & -0.0005 & -0.0157 & 0.28 & -2.3206 & -1.123 \\
\hline 0.3026 & 0.7748 & 1.391 & 1191 & 1.6560 & -0.0006 & -0.0220 & 0.36 & -2.3740 & -1.256 \\
\hline 0.4031 & 0.7684 & 1.392 & 1195 & 1.4045 & -0.0007 & -0.0256 & 0.41 & -2.1260 & -1.080 \\
\hline 0.5032 & 0.7622 & 1.392 & 1198 & 1.2752 & -0.0007 & -0.0265 & 0.43 & -1.7563 & -0.735 \\
\hline 0.6029 & 0.7561 & 1.393 & 1202 & 1.1065 & -0.0007 & -0.0249 & 0.43 & -1.4268 & -0.506 \\
\hline 0.7026 & 0.7500 & 1.393 & 1205 & 0.9783 & -0.0006 & -0.0209 & 0.36 & -1.0555 & -0.230 \\
\hline 0.7980 & 0.7443 & 1.394 & 1208 & 0.8325 & -0.0005 & -0.0147 & 0.27 & -0.7221 & -0.077 \\
\hline 0.9012 & 0.7383 & 1.395 & 1212 & 0.6678 & -0.0003 & -0.0057 & 0.15 & -0.3670 & -0.025 \\
\hline 1.0000 & 0.7325 & 1.395 & 1215 & 0.5358 & 0.0000 & 0.0000 & 0.00 & 0.0000 & 0.000 \\
\hline \multicolumn{10}{|c|}{$T=313 \mathrm{~K}$} \\
\hline 0.0000 & 0.7871 & 1.385 & 1141 & 4.7960 & 0.0000 & 0.0000 & 0.00 & 0.0000 & 0.000 \\
\hline 0.1012 & 0.7802 & 1.386 & 1142 & 2.9805 & -0.0004 & -0.0086 & 0.18 & -1.3513 & -0.625 \\
\hline 0.2015 & 0.7734 & 1.387 & 1143 & 2.2640 & -0.0007 & -0.0189 & 0.30 & -1.6139 & -0.733 \\
\hline 0.3026 & 0.7666 & 1.387 & 1144 & 1.6765 & -0.0009 & -0.0261 & 0.39 & -1.7499 & -0.902 \\
\hline 0.4031 & 0.7600 & 1.388 & 1145 & 1.5416 & -0.0010 & -0.0302 & 0.46 & -1.4419 & -0.512 \\
\hline 0.5032 & 0.7535 & 1.389 & 1146 & 1.3784 & -0.0011 & -0.0313 & 0.49 & -1.1697 & -0.197 \\
\hline 0.6029 & 0.7472 & 1.389 & 1147 & 1.2170 & -0.0010 & -0.0295 & 0.50 & -0.9031 & 0.082 \\
\hline 0.7026 & 0.7409 & 1.390 & 1148 & 0.9894 & -0.0009 & -0.0248 & 0.41 & -0.7080 & 0.147 \\
\hline 0.7980 & 0.7350 & 1.391 & 1149 & 0.8258 & -0.0007 & -0.0177 & 0.30 & -0.4724 & 0.253 \\
\hline 0.9012 & 0.7287 & 1.391 & 1149 & 0.6088 & -0.0004 & -0.0073 & 0.17 & -0.2629 & 0.084 \\
\hline 1.0000 & 0.7227 & 1.392 & 1150 & 0.4685 & 0.0000 & 0.0000 & 0.00 & 0.0000 & 0.000 \\
\hline
\end{tabular}

$$
R_{\mathrm{m}}^{\text {expt }}=\frac{n_{\mathrm{m}}^{2}-1}{n_{\mathrm{m}}^{2}+2} \frac{x_{1} M_{1}+x_{2} M_{2}}{\rho_{\mathrm{m}}},
$$

and

$$
R_{\mathrm{m}}^{\mathrm{id}}=\frac{n_{1}^{2}-1}{n_{1}^{2}+2} \frac{M_{1}}{\rho_{1}} \phi_{1}+\frac{n_{2}^{2}-1}{n_{2}^{2}+2} \frac{M_{2}}{\rho_{2}} \phi_{2},
$$




$$
\begin{aligned}
& \Delta u=u-\left(x_{1} u_{1}+x_{2} u_{2}\right), \\
& \Delta \eta=\eta-\left(x_{1} \eta_{1}+x_{2} \eta_{2}\right), \\
& \Delta G^{* \mathrm{E}}=R T\left[\ln \left(\frac{\eta V_{\mathrm{m}}}{\eta_{2} V_{\mathrm{m} 2}}\right)-x_{1} \ln \left(\frac{\eta_{1} V_{\mathrm{m} 1}}{\eta_{2} V_{\mathrm{m} 2}}\right)\right] .
\end{aligned}
$$

Mixing rules for refractive index $[10,11]$ are listed below.

The Lorentz-Lorentz relation for refractive index is based on the change in the molecular polarizability with volume fraction

$$
\frac{n_{\mathrm{m}}^{2}-1}{n_{\mathrm{m}}^{2}+2} \frac{1}{\rho_{\mathrm{m}}}=\frac{n_{1}^{2}-1}{n_{1}^{2}+2} \frac{w_{1}}{\rho_{1}}+\frac{n_{2}^{2}-1}{n_{2}^{2}+2} \frac{w_{2}}{\rho_{2}} .
$$

Gladstone-Dale equation for predicting the refractive index of a binary mixture is as follows:

$$
n_{\mathrm{m}}-1=\varphi_{1}\left(n_{1}-1\right)+\varphi_{2}\left(n_{2}-1\right) .
$$

Wiener's relation may be represented as

$$
\frac{n_{\mathrm{m}}^{2}-n_{1}^{2}}{n_{\mathrm{m}}^{2}+2 n_{1}^{2}}=\frac{n_{2}^{2}-n_{1}^{2}}{n_{2}^{2}+2 n_{1}^{2}} \varphi_{2} .
$$

Heller's relation is given by

$$
\frac{n_{\mathrm{m}}-n_{1}}{n_{1}}=\frac{3}{2} \frac{m^{2}-1}{m^{2}+2} \varphi_{2}
$$

Arago-Biot, assuming volume additivity, proposed the following relation for refractive index of binary mixtures:

$$
n_{\mathrm{m}}=\varphi_{1} n_{1}+\varphi_{2} n_{2} .
$$

Newton gave the following equation:

$$
n_{\mathrm{m}}^{2}-1=\varphi\left(n_{1}^{2}-1\right)+\varphi_{2}\left(n_{2}^{2}-1\right) .
$$

Eykman's relation may be represented as

$$
\frac{n_{\mathrm{m}}^{2}-1}{n_{\mathrm{m}}+0.4} V_{\mathrm{m}}=\frac{n_{1}^{2}-1}{n_{1}+0.4} \frac{M_{1} x_{1}}{\rho_{1}}+\frac{n_{2}^{2}-1}{n_{2}+0.4} \frac{M_{2} x_{2}}{\rho_{2}} .
$$

In Eqs. (1)-(12) $M_{i}, \rho_{i}, n_{i}, u_{i}, \eta_{i}, \phi_{i}, V_{\mathrm{m} i}, W_{i}$, and $x_{i}$, respectively, represent molecular weight, density, refractive index, ultrasonic velocity, viscosity, volume fraction, molar volume, weight fraction, and mole fraction of the $i$-th component ( $i=1,2$ for binary mixtures), $\rho_{\mathrm{m}}, n_{\mathrm{m}}, u, \eta$, and $V_{\mathrm{m}}$ are density, refractive index, ultrasonic velocity, viscosity, and molar volume of the mixtures. $R$ is the universal gas constant and $T$ is the absolute temperature. All the excess parameters are summarized in Tables I and II for butylamine + 1-butanol, butylamine + tert-butanol mixtures and are shown graphically in Figs. 1 to 5 .

The values of $V_{\mathrm{m}}^{\mathrm{E}}, \Delta R_{\mathrm{m}}, \Delta u, \Delta \eta$, and $\Delta G^{* \mathrm{E}}$ for each mixture have been fitted to Redlich-Kister polynomial equation

$$
Y^{\mathrm{E}}=x(1-x) \sum_{i=1}^{5} a_{i}(1-2 x)^{i-1},
$$



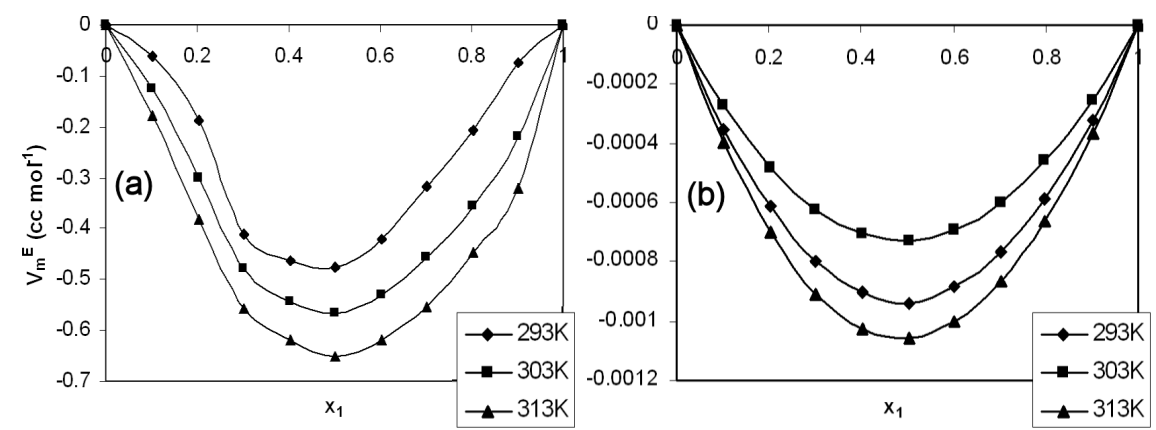

Fig. 1. (a) Excess molar volume $V_{\mathrm{m}}^{\mathrm{E}}$ as a function of butylamine $\left(x_{1}\right)$ for butylamine +1 -butanol mixture at all the three temperatures. (b) As in (a), but for butylamine + tert-butanol mixture.
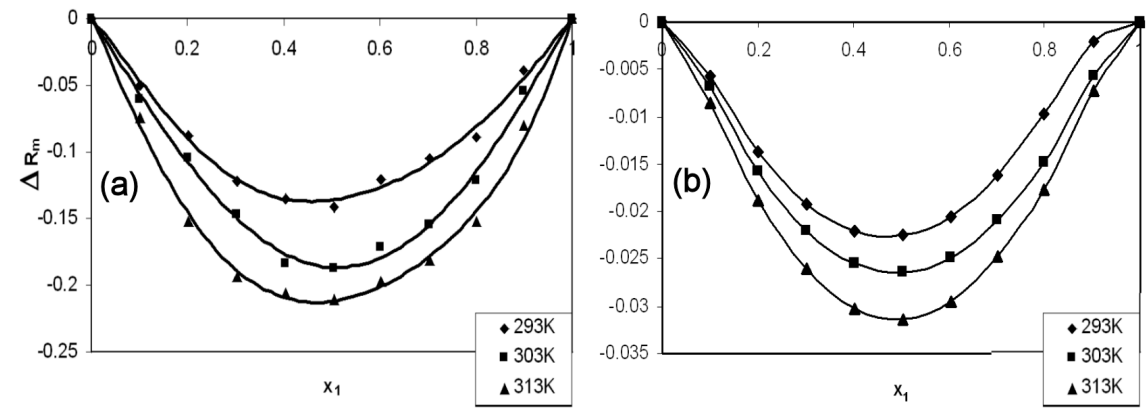

Fig. 2. (a) Molar refraction deviation $\Delta R_{\mathrm{m}}$ as a function of butylamine $\left(x_{1}\right)$ for butylamine +1 -butanol mixture at all the three temperatures. (b) As in (a), but for butylamine + tert-butanol mixture.
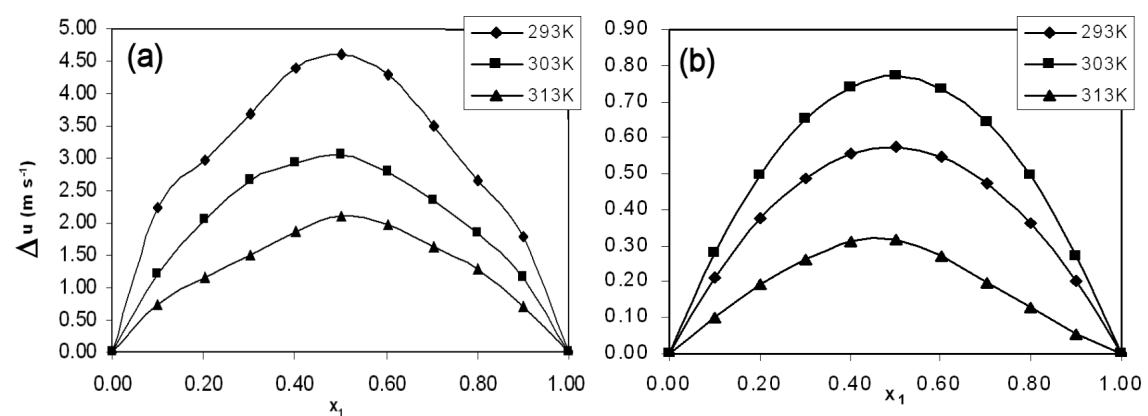

Fig. 3. (a) Deviation in ultrasonic velocity $\Delta u$ as a function of butylamine $\left(x_{1}\right)$ for butylamine + 1-butanol mixture at all the three temperatures. (b) As in (a), but for butylamine + tert-butanol mixture. 

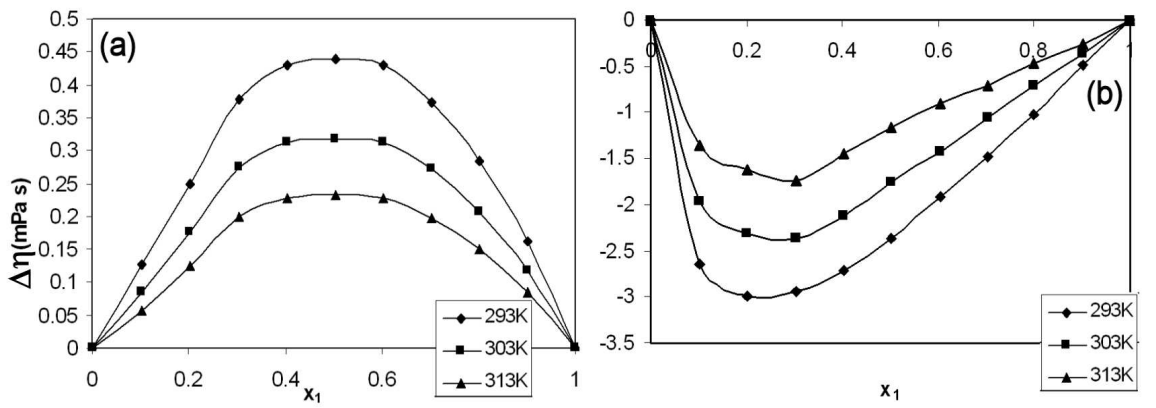

Fig. 4. (a) Viscosity deviation $\Delta \eta$ as a function of butylamine $\left(x_{1}\right)$ for butylamine + 1-butanol mixture at all the three temperatures. (b) As in (a), but for butylamine + tert-butanol mixture.
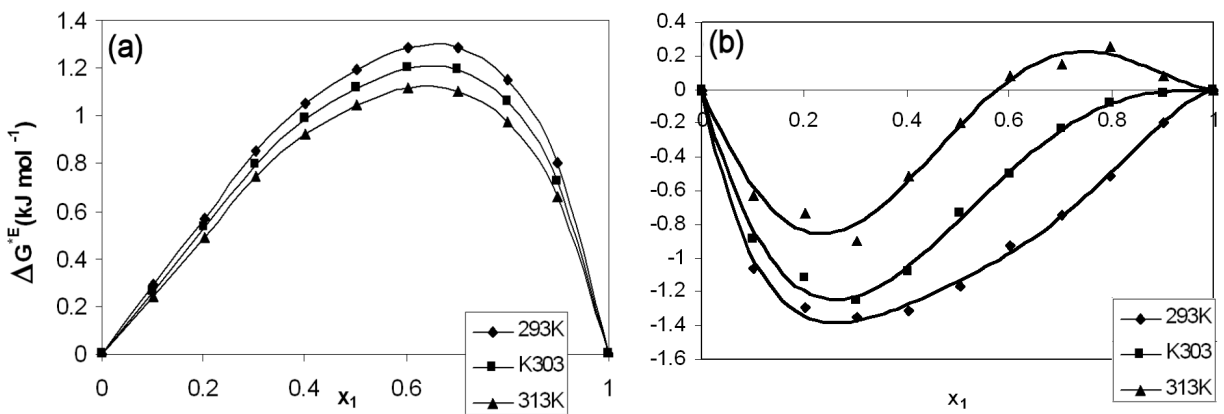

Fig. 5. Excess Gibb's free energy of activation for viscous flow $\Delta G^{* \mathrm{E}}$ as a function of butylamine $\left(x_{1}\right)$ for butylamine +1 -butanol mixture at all the three temperatures. (b) As in (a), but for butylamine + tert-butanol mixture.

where $Y^{\mathrm{E}}$ refers to excess/deviation parameter. The values of the coefficients $a_{i}$ were calculated by the method of least squares along with the standard deviation $\sigma\left(Y^{\mathrm{E}}\right)$, which are given in Table III. The coefficient $i$ is an adjustable parameter for the best fit of the excess functions.

The standard deviation values were obtained from

$$
\sigma\left(Y^{\mathrm{E}}\right)=\left[\frac{\sum\left(Y_{\text {expt }}-Y_{\text {cal }}\right)}{n-p}\right]^{1 / 2},
$$

where $n$ is the total number of experimental points, $p$ is the number of coefficients, $Y_{\text {expt }}$ and $Y_{\text {cal }}$ are the experimental and calculated excess parameters, respectively.

According to Fort and Moore [12], a negative excess molar volume $V_{\mathrm{m}}^{\mathrm{E}}$ is an indication of strong heteromolecular interaction in the liquid mixtures and is attributed to change-transfer, dipole-dipole interactions, and hydrogen bonding between the unlike molecules, while a positive sign indicates a weak interaction and is attributed to dispersive forces. The magnitudes of the contribution will vary 
TABLE III

Adjustable parameters $a_{i}$ with the standard deviations $\sigma\left(Y^{\mathrm{E}}\right)$ for excess molar volume $V_{\mathrm{m}}^{\mathrm{E}}$, molar refraction deviation $\Delta R_{\mathrm{m}}$, deviation in ultrasonic velocity $\Delta u$, viscosity deviation $\Delta \eta$, and excess Gibb's free energy of activation for viscous flow $\Delta G^{* \mathrm{E}}$ for both the binary mixtures at temperature $T$.

\begin{tabular}{|c|c|c|c|c|c|c|}
\hline Functions & $a_{1}$ & $a_{2}$ & $a_{3}$ & $a_{4}$ & $a_{5}$ & $\sigma\left(Y^{\mathrm{E}}\right)$ \\
\hline \multicolumn{7}{|c|}{ butylamine + 1-butanol } \\
\hline \multicolumn{7}{|c|}{$T=293 \mathrm{~K}$} \\
\hline$V_{\mathrm{m}}^{\mathrm{E}}\left[\mathrm{cc} \mathrm{mol} \mathrm{mol}^{-1}\right]$ & -2.7352 & 0.1091 & 5.7878 & 0.0100 & -4.2354 & 0.0185 \\
\hline$\Delta R_{\mathrm{m}}$ & -0.3075 & 0.1167 & -1.2200 & -0.2893 & 1.4631 & 0.0026 \\
\hline$\Delta u\left[\mathrm{~m} \mathrm{~s}^{-1}\right]$ & 2.8128 & 0.6734 & -3.0512 & -0.6426 & 2.4812 & 0.0101 \\
\hline$\Delta \eta[\mathrm{mPa} \mathrm{s}]$ & 1.7588 & 1.3502 & -2.8873 & -2.4902 & 4.1265 & 0.0062 \\
\hline$\Delta G^{* \mathrm{E}}\left[\mathrm{kJ} \mathrm{mol}^{-1}\right]$ & 4.7663 & -1.2483 & -0.8438 & -3.6428 & 4.4870 & 0.0062 \\
\hline \multicolumn{7}{|c|}{$T=303 \mathrm{~K}$} \\
\hline$V_{\mathrm{m}}^{\mathrm{E}}\left[\mathrm{cc} \mathrm{mol}^{-1}\right]$ & -2.2418 & 0.1183 & 0.7154 & 1.1997 & -0.3612 & 0.0088 \\
\hline$\Delta R_{\mathrm{m}}$ & -0.7517 & -0.6084 & 1.4337 & 0.8919 & 2.5799 & 0.0031 \\
\hline$\Delta u\left[\mathrm{~m} \mathrm{~s}^{-1}\right]$ & 2.8396 & 0.0039 & -0.6800 & -0.0314 & 1.9891 & 0.0119 \\
\hline$\Delta \eta[\mathrm{mPa} \mathrm{s}]$ & 1.2756 & 1.0935 & -2.3223 & -2.0557 & 3.2663 & 0.0051 \\
\hline$\Delta G^{* \mathrm{E}}\left[\mathrm{kJ} \mathrm{mol}^{-1}\right]$ & 4.4550 & -0.8614 & -1.4084 & -3.7104 & 4.7677 & 0.0067 \\
\hline \multicolumn{7}{|c|}{$T=313 \mathrm{~K}$} \\
\hline$V_{\mathrm{m}}^{\mathrm{E}}\left[\mathrm{cc} \mathrm{mol}^{-1}\right]$ & -2.6484 & -0.3158 & 0.4029 & 1.8694 & 0.6349 & 0.0079 \\
\hline$\Delta R_{\mathrm{m}}$ & -0.8453 & -0.9300 & -1.4544 & 6.2933 & 8.2021 & 0.0416 \\
\hline$\Delta u\left[\mathrm{~m} \mathrm{~s}^{-1}\right]$ & 3.6083 & -0.6669 & 0.6641 & 0.8993 & 2.7189 & 0.0076 \\
\hline$\Delta \eta[\mathrm{mPa} \mathrm{s}]$ & 0.9348 & 0.8450 & -1.8077 & -1.6347 & 2.4777 & 0.0040 \\
\hline$\Delta G^{* \mathrm{E}}\left[\mathrm{kJ} \mathrm{mol}^{-1}\right]$ & 4.1697 & -0.7172 & -1.5392 & -3.4585 & 4.4195 & 0.0062 \\
\hline \multicolumn{7}{|c|}{ butylamine + tert-butanol } \\
\hline \multicolumn{7}{|c|}{$T=293 \mathrm{~K}$} \\
\hline$V_{\mathrm{m}}^{\mathrm{E}}\left[\mathrm{cc} \mathrm{mol}^{-1}\right]$ & -0.0038 & 0.0000 & 0.0004 & -0.0001 & -0.0004 & 0.0000 \\
\hline$\Delta R_{\mathrm{m}}$ & -0.0933 & 0.0071 & -0.0057 & -0.0492 & 0.1289 & 0.0001 \\
\hline$\Delta u\left[\mathrm{~m} \mathrm{~s}^{-1}\right]$ & 1.2959 & -0.2210 & 0.6007 & 0.3178 & -1.1162 & 0.0047 \\
\hline$\Delta \eta[\mathrm{mPa} \mathrm{s}]$ & -9.3427 & -8.2706 & -9.4929 & -10.3303 & -4.9512 & 0.0307 \\
\hline$\Delta G^{* \mathrm{E}}\left[\mathrm{kJ} \mathrm{mol}^{-1}\right]$ & -4.5957 & -3.5642 & -4.5549 & -3.6679 & 1.2399 & 0.0182 \\
\hline \multicolumn{7}{|c|}{$T=303 \mathrm{~K}$} \\
\hline$V_{\mathrm{m}}^{\mathrm{E}}\left[\mathrm{cc} \mathrm{mol}^{-1}\right]$ & -0.0029 & -0.0002 & 0.0003 & 0.0001 & -0.0002 & 0.0000 \\
\hline$\Delta R_{\mathrm{m}}$ & -0.1091 & 0.0148 & -0.0133 & -0.0326 & 0.1170 & 0.0001 \\
\hline$\Delta u\left[\mathrm{~m} \mathrm{~s}^{-1}\right]$ & 1.8271 & -0.9722 & 1.6243 & 1.3659 & -3.0403 & 0.0050 \\
\hline$\Delta \eta[\mathrm{mPa} \mathrm{s}]$ & -7.1154 & -13.3623 & 10.9313 & 3.6904 & -31.1975 & 0.0363 \\
\hline$\Delta G^{* \mathrm{E}}\left[\mathrm{kJ} \mathrm{mol}^{-1}\right]$ & -3.5474 & -5.2484 & 1.8237 & -1.0549 & -6.4960 & 0.0384 \\
\hline \multicolumn{7}{|c|}{$T=313 \mathrm{~K}$} \\
\hline$V_{\mathrm{m}}^{\mathrm{E}}\left[\mathrm{cc} \mathrm{mol}^{-1}\right]$ & -0.0042 & -0.0006 & 0.0005 & 0.0006 & -0.0009 & 0.0000 \\
\hline$\Delta R_{\mathrm{m}}$ & -0.1249 & 0.0031 & -0.0116 & -0.0162 & 0.1068 & 0.0001 \\
\hline$\Delta u\left[\mathrm{~m} \mathrm{~s}^{-1}\right]$ & 1.8503 & -0.0335 & -0.0769 & 0.1226 & 0.3475 & 0.0069 \\
\hline$\Delta \eta[\mathrm{mPa} \mathrm{s}]$ & -4.4301 & -4.5759 & -3.8543 & -4.4739 & -4.9215 & 0.0569 \\
\hline$\Delta G^{* \mathrm{E}}\left[\mathrm{kJ} \mathrm{mol}^{-1}\right]$ & -0.7821 & -6.330 & -0.2592 & 2.3868 & -4.8700 & 0.0736 \\
\hline
\end{tabular}


with the components and composition of the mixtures. In the present investigation, both the mixtures gave a negative magnitude of $V_{\mathrm{m}}^{\mathrm{E}}$, and they depict the presence of heteromolecular interaction, which support the Fort and Moore [12] observation. Wen-Lu Weng [13] and Papaioannou et al. [14] have also observed negative values of $V_{\mathrm{m}}^{\mathrm{E}}$ in the case of butylamine +1 -butanol mixture. The negative $V_{\mathrm{m}}^{\mathrm{E}}$ values of butylamine +1 -butanol observed in the present investigation have been found to be in good agreement with the values of $V_{\mathrm{m}}^{\mathrm{E}}$ reported earlier by Wen-Lu Weng [13] and Papaioannou et al. [14]. The negative values of $V_{\mathrm{m}}^{\mathrm{E}}$ indicate that volume contraction takes place upon mixing butylamine with 1-butanol/tert-butanol due to cross association between these dissimilar molecules [15]. The magnitude of the volume contraction follows the sequence of 1-butanol $>$ tert-butanol. This is in agreement with the results reported by Ali and Nain [15] on binary mixtures of tetrahydrofuran (THF) with 1-butanol and tert-butanol which suggest that the excess values follow the same sequence, due to the steric hindrance produced by the presence of three methyl groups at $3^{0}$ carbon atom in tert-butanol, as compared to one butyl group in 1-butanol. Molar refraction deviation $\Delta R_{\mathrm{m}}$ as calculated from Eq. (2b) represents the electronic perturbation due to orbital mixing of two components [16]. $R_{\mathrm{m}}$ gives the strength of the interaction in mixture and is a sensitive function of wavelength, temperature, and mixture composition. Figure 2 shows that $\Delta R_{\mathrm{m}}$ values are negative for both the mixtures at all the temperatures. The values of $V_{\mathrm{m}}^{\mathrm{E}}$ and $\Delta R_{\mathrm{m}}$ support each other. The $V_{\mathrm{m}}^{\mathrm{E}}$ and $\Delta R_{\mathrm{m}}$ values become more negative at higher temperature for both the mixtures, suggesting an increase in interaction between unlike molecules.

Deviation in ultrasonic velocity $\Delta u$, viscosity deviation $\Delta \eta$, and excess Gibb's free energy of activation for viscous flow $\Delta G^{* \mathrm{E}}$ can be used to detect the molecular interactions present in the binary mixture. $\Delta u$ has been found to be positive for both the mixtures over the entire mole fraction range (Fig. 3). It is evident from Fig. 3 that in both the mixtures containing butylamine, $\Delta u$ increases as a function of mole fraction of butylamine, attains maxima and then decreases. The values of $\Delta u$ systematically become more positive with the rise in temperature suggesting that specific interaction increases due to enhanced thermal energy.

Figure 4 shows that viscosity deviation $\Delta \eta$ values are positive for butylamine + 1-butanol and negative for butylamine + tert-butanol mixtures. The positive values of $\Delta n$ in butylamine +1 -butanol mixture can be explained on the basis of complex formation between unlike molecules through hydrogen bonding. The negative values of $\Delta n$ for butylamine + tert-butanol may be attributed to the presence of weak dipole-dipole interaction due to predominance rupture of dipolar association of tert-butanol and steric hindrance produced by three methyl groups over the hydrogen bond formed between unlike molecules. Our positive values of $\Delta \eta$ in the case of butylamine +1 -butanol do not corroborate with the values of $\Delta \eta$ on the same mixtures reported earlier by Wen-Lu Weng [13] and Dominguez et al. [17]. However, all the excess parameters $V_{\mathrm{m}}^{\mathrm{E}}, \Delta R_{\mathrm{m}}$, and $\Delta \eta$ reported 
here by us are in conformity with each other. $\Delta \eta$ values are found to decrease with increasing temperature in the positive and negative directions in the cases of butylamine +1 -butanol and butylamine + tert-butanol mixtures, respectively.

Figure 5 shows that $\Delta G^{* \mathrm{E}}$ values are positive for butylamine +1 -butanol and negative for butylamine + tert-butanol mixtures, respectively. However, a smaller positive trend has been observed at a temperature of $313 \mathrm{~K}$ in butylamine rich region. The positive values of $\Delta G^{* \mathrm{E}}$ indicates the presence of strong interaction in butylamine + 1-butanol mixture, whereas negative $\Delta G^{* \mathrm{E}}$ values for butylamine + tert-butanol mixture may be assigned to the predominance of dispersive forces and also due to less acidic nature of tertiary alcohols than primary alcohols [18]. Gupta et al. [19] and Mialkowski et al. [20] have also reported similar variations in the $\Delta G^{* \mathrm{E}}$ values for binary mixtures of ether + alcohols and $\gamma$-butyrolactone + dimethylcarbonate.

TABLE IV

APD of theoretically calculated refractive indices from different semi-empirical relations at all the three temperatures.

\begin{tabular}{c|c|c|c|c|c|c|c}
\hline \hline $\begin{array}{c}\text { Temp. } \\
{[\mathrm{K}]}\end{array}$ & L-L & G-D & W & H & A-B & N & Eyk \\
\hline \multicolumn{7}{c}{ butylamine + 1-butanol } \\
\hline 293 & -0.0786 & 0.00278 & 0.00280 & 0.00285 & 0.00279 & 0.00272 & -0.07384 \\
303 & -0.11447 & -0.00617 & -0.00615 & -0.00613 & -0.00617 & -0.06620 & -0.10786 \\
313 & -0.1415 & -0.01283 & -0.01282 & -0.01279 & -0.01283 & -0.01287 & -0.13357 \\
\hline \multicolumn{7}{c}{ butylamine + tert-butanol } \\
\hline 293 & 0.00799 & 0.00800 & 0.00806 & 0.00820 & 0.00800 & 0.00781 & 0.00788 \\
303 & 0.00587 & 0.00588 & 0.00593 & 0.00604 & 0.00589 & 0.00574 & 0.00579 \\
313 & 0.00589 & 0.00591 & 0.00598 & 0.00612 & 0.00591 & 0.00592 & 0.00578
\end{tabular}

Further, seven empirical relations (Eqs. (6) to (12)) were used for the prediction of refractive indices. The refractive index data correlated with these semi-empirical equations for both the binary mixtures show an excellent agreement with the respective experimental values of refractive index (Table IV). It can be seen from Table IV that the correlation of these equations showed the suitability of all the seven relations for representing the mixing refractive indices of the binary mixtures of butylamine +1 -butanol/tert-butanol. The applicability of these semi-empirical relations for predicting refractive index has also been emphasized by others $[10,11]$.

\section{Conclusions}

The observed negative and positive values of various excess parameters for butylamine +1 -butanol system are attributed to the strong intermolecular inter- 
action through H-bonding. The less negative values of $V_{\mathrm{m}}^{\mathrm{E}}$ and $\Delta R_{\mathrm{m}}$ at all the three temperatures and small positive values of $\Delta G^{* \mathrm{E}}$ at $313 \mathrm{~K}$ for binary mixture of butylamine + tert-butanol are due to the weak specific interactions arising from induced dipole-dipole forces. However, dispersive forces may be responsible for negative values of $\Delta \eta$.

\section{References}

[1] A. Ali, A.K. Nain, V.K. Sharma, S. Ahmad, Ind. J. Phys. 75B, 519 (2001).

[2] A. Pal, H. Kumar, Ind. J. Phys. 75B, 419 (2001).

[3] A. Ali, H. Soghra, Ind. J. Phys. 76B, 23 (2002).

[4] A. Pal, H. Kumar, Fluid Phase Equilib. 181, 17 (2001).

[5] T.M. Letcher, G.G. Redhi, Fluid Phase Equilib. 198, 257 (2002).

[6] H. Iloukhani, K. Khanlarzadeh, J. Chem. Eng. Data 51, 1226 (2006).

[7] J.M. Resa, S. Gonzalez, O.D. Zandaluce, J. Lanz, J. Chem. Eng. Data 24, 995 (2002).

[8] CRC Handbook of Chemistry and Physics, Ed. D.R. Lide, 76th ed., CRC Press, India 1995-1996, p. 32.

[9] D.D. Perrin, W.L.F. Armarego, Purification of Laboratory Chemicals, Pergamon Press, Oxford 1980.

[10] J.D. Pandey, A.K. Shukla, R.K. Shukla, R.D. Rai, Ind. J. Chem. 27A, 336 (1988).

[11] T.M. Aminabhavi, J. Chem. Eng. Data 29, 54 (1984).

[12] R.J. Fort, W.R. Moore, Trans. Faradays Soc. 61, 2102 (1965).

[13] Wen-Lu Weng, J. Chem. Eng. Data 45, 606 (2000).

[14] D. Papaioannou, M. Bridakis, C.G. Panayiotou, J. Chem. Eng. Data 38, 370 (1993).

[15] A. Ali, A.K. Nain, Pramana J. Phys. 58, 695 (2002).

[16] M. Gupta, I. Vibhu, J.P. Shukla, J. Phys. Chem. Liq. 41, 575 (2003).

[17] M. Dominguez, J. Pardo, M.C. Lopez, F.M. Royo, J.S. Urieta, Fluid Phase Equilib. 124, 147 (1996).

[18] R.J. Morrison, R.N. Boyd, Organic Chemistry, 6th ed., Prentice Hall of India, Pvt. India 1999.

[19] M. Gupta, I. Vibhu, J.P. Shukla, Fluid Phase Equilib. 244, 26 (2006).

[20] C. Mialkowski, A. Chagnes, B. Carré, D. Willmann, D. Lemordant, J. Chem. Thermodyn. 34, 1847 (2002). 